\title{
Estilos de afrontamiento y el bienestar psicológico de los alumnos de Psicología de la Universidad Católica de Santa María
}

\author{
Coping Styles and the Psychological Well-being of the Psychology Students \\ of the Catholic University of Santa María \\ Andrea Quintanilla Acosta \\ Universidad Católica de Santa María, Arequipa, Perú \\ (iD) https://orcid.org/oooo-0oo2-7471-5610 \\ Correspondencia: quintanillaandrea26@gmail.com
}

\begin{abstract}
Resumen
En la actualidad, hablar de estrés es muy común y es importante estudiar las formas de afrontarlo, por eso nuestro objetivo es establecer el tipo de relación entre los estilos de afrontamiento y el bienestar psicológico de los alumnos de psicología de la Universidad Católica de Santa María en Arequipa. Para ello se hizo un estudio correlacional con 120 participantes a los que se les aplicó el Cuestionario de Afrontamiento al Estrés (CAE) y la Escala de Bienestar Psicológico (BIEPS). Para obtener los resultados se aplicó la correlación de Pearson obteniendo que los estudiantes de psicologia que autoperciben un mayor bienestar psicológico utilizan técnicas de afrontamiento de focalización en la solución del problema, revaluación positiva y búsqueda de apoyo social, ya que presentan correlaciones significativas positivas moderadas. Entonces las personas con un elevado bienestar se caracterizaron por analizar la causa de los problemas de un modo sistemático, ver el lado positivo y pedir ayuda. Palabras clave: Estilos de afrontamiento, bienestar psicológico, estrés.
\end{abstract}

\begin{abstract}
Currently stress is a very common topic and it is considered useful to study ways to manage stress. Our goal, then, was to establish the relationship between coping styles and the psychological well-being of psychology students at the Catholic University of Santa María in Arequipa. For this purpose, a correlational study was conducted with 120 participants to whom the Stress Coping Questionnaire (CAE) and the Psychological Wellbeing Scale (BIEPS) were applied. To analyze the results, a Pearson correlation was calculated. Positive significant correlations indicated that psychology students who perceive greater psychological
\end{abstract}


well-being used coping techniques of focusing on the solution of the problem, positive revaluation and the search for social support. Those students who rated themselves as having a high level of well-being were characterized by analyzing the cause of the problems in a systematic way, seeing the positive side and asking for help.

Keywords: Coping styles, psychological well-being, stress.

\section{Introducción}

En la actualidad, hablar de estrés es muy común, y ante cualquier situación problemática se refieren altos niveles de estrés; pero no todos perciben el estrés de la misma forma ni intensidad, esto es debidoa los diferentes estilos de afrontamiento que posee cada persona. Asimismo, se observa que no todas las personas disfrutan de un buen bienestar psicológico, sintiéndose muchas de ellas, insatisfechas con sus logros y lo que poseen, lo que al final trae muchas consecuencias en el desarrollo y plenitud de la vida (Urbano, 2019).

La Organización Mundial de la Salud (2013), informó que más de 88 millones de personas en América Latina y el Caribe poseen trastornos afectivos desencadenados por el estrés. Siendo las personas que estudian las que presentan altos niveles del mismo. En el informe Stress America presentado por la American Psychological Association (APA), en el año 2013 el 39\% de las personas entre 18 y 33 años se declararon estresadas y el $60 \%$ de estudiantes universitarios dijieron presentar niveles altos de estrés.

Lazarus y Folkman (1984) señalan que existen diversas estrategias para tratar con el estrés, las cuales pueden ser o no adaptativas. Ellos definen el afrontamiento como el uso de variados recursos, de nivel cognitivo, conductual y emocional, encargados de reducir o hacer tolerable las exigencias que generan estrés. Es importante recordar que Díaz (2010) concluyó que el afrontamiento no es sinónimo de bienestar, ya que en determinados casos puede funcionar y en otros no, debido a que es un proceso dinámico, que se ajusta al contexto y las demandas que se le presentan, asi como de su clasificacion.

Existen dos estilos tradicionales para afrontar el estrés: centrado en el problema y centrado en la emoción (Macías et al., 2013). Sin embargo, hay otros estilos como los propuestos por Sandín y Chorot (2003), los cuales comprenden la búsqueda de apoyo social (pedir consejo), la expresión emocional abierta (descargar el mal humor con los demás de manera agresiva), u hostil, la religión (ayuda espiritual), el focalizado en la solución del problema (análisis de las causas), la autofocalización negativa (autonconvencerse negativamente de que las cosas siempre salen mal), la evitación (enfocarse en otras actividades para olvidar el problema) y reevaluación positiva (sacar lo mejor de una situación).

Una de las variables que más afecta el estrés es el bienestar psicolgico, trayendo sentimientos de inadecuación y de 
insatisfacción de uno mismo (Gómez et al., 2007). Casulloy Castro (2000) definen el bienestar como la percepción personal de la vida de manera global y positiva. En palabras más sencillas es el cómo las personas se sienten satisfechas con su vida en términos generales. Se dice que se tiene un alto bienestar si hay satisfacción con la vida y reiteradas emociones positivas. $\mathrm{Si}$ por el contrario, se expresa insatisfacción y pocas emociones positivas, su bienestar se consideraría bajo (Omar et al., 2009). Algunas investigaciones revelan que las personas más contentas y satisfechas con la vida experimentan menos estrés (Gómez et al., 2007).

Según la teoría de la autodeterminacion del bienestar psicológico referida por Ryan y Deci (2000, citados por Cuadra \& Florenzano, 2003), se dice que el contexto ambiental donde las personas se desarrollan y creen, promueve u obstaculiza los procesos positivos de los estilos de afrontamiento; en ese sentido, el contexto social es determinante para un desarrollo y funcionamiento exitoso. Por el contrario, entornos que no contribuyen en el soporte generan enajenación y enfermedad en el sujeto. Hay que entender que las personas que poseen estrategias de afrontamiento adaptativas tienen mayor capacidad para cumpliry lograr retos cada vez mayores, lo que favorecería el impacto positivo en el bienestar subjetivo del individuo (Cuadra \& Florenzano, 2003).

González, Montoya, Casullo y Bernabéu (2002) encontraron una relación significativa entre ambas variables, siendo las mujeres las que poseen más estrategias de afrontamiento y menos habilidad para afrontar los problemas que los varones. Quiros (2013), en un estudio sobre el afrontamiento en agentes del tráfico aéreo, encontró que el estilo de afrontamiento más usado es el enfocado en el problema, seguido por la emoción y, finalmente, el evitativo. En la misma investigación se observó que la reinterpretación positiva, la planificación, el afrontamiento activo y la búsqueda de soporte social, fueron las estrategias de afrontamiento más utilizadas. Vallejo (2007) igualmente identificó relación altamente significativa entre las variables estudiadas. Matalinares, Díaz, Ornella, Baca, Uceda y Yaringaño (2016) evidenciaron que existe una relación directa y significativa entre el modo de afrontamiento dirigido al problema y el modo de afrontamiento dirigido a la emoción con la variable bienestar psicológico. Asimismo, se encontró una relación significativa pero indirecta entre otros estilos de afrontamiento y el bienestar psicológico. Urrelo y Huamani (2019) encontraron correlaciones significativas entre la satisfacción con la viday los estilos de afrontamiento; de modo que $71.8 \%$ de la población se encuentra en un rango de satisfecha y muy satisfecha, siendo los varones quienes presentan una mayor satisfacción, y quienes tienen 18 años. Tacca y Tacca (2019) realizarón una investigación sobre estilos de afrontamiento y bienestar psicológico en estudiantes de postgrado encontrando que el estilo de afrontamiento centrado en el problema y el estilo centrado en las emociones se relacionan positivamente con el bienestar psicológico respectivamente. Además, se encontró que los estudiantes de más de 
41 años emplean una gama más amplia de estrategias de afrontamiento y presentan mayor nivel de bienestar psicológico.

En base a los hallazgos anteriores, y viendo la creciente importancia en estos temas es que pretendemos establecer el tipo de relación entre los estilos de afrontamiento y el bienestar psicológico de los alumnos de psicología de la Universidad Católica de Santa María en Arequipa. El presente trabajo tiene un potencial impacto teórico ya que aporta nuevo conocimiento para mejorar y potenciar las estrategias de afrontamiento para crear un adecuado nivel de bienestar psicológico, y nos permite ampliar conceptos relacionados al tema. El potencial impacto práctico es que podemos usar esta informacion para crear y mejorar la eficiencia y eficacia de programas sobre el manejo del estrés y el bienestar, y prevenir la aparición de emociones negativas entre los estudiantes universitarios. Es imporatnte trabajar en este tema ya que se ha evidenciado que entre el afrontamiento y el bienestar psicológico existe una relación positiva y significativa (Romero et al., 2010) y que una adecuada estrategia de afrontamiento contribuye significativamente a lograr un alto nivel de percepcion de satisfaccion con la vida (Cornejo \& Lucero, 2005), mientras que elevados niveles de bienestar se asocian al esfuerzo, la resolución de problemas y el manejo de emociones para reducir los momentos de estrés (Marsollier \& Aparicio, 2011).
Nuestra pregunta de investigación es por tanto: ¿Qué relación existe entre los estilos de afrontamiento y el bienestar psicológico de los alumnos de psicología de la Universidad Católica de Santa María en Arequipa?

\section{Método}

\section{Tipo y diseño de investigación}

Se realizó un estudio no experimental de alcance correlacional, ya que no hay manipulacion intencionada de variables y solo se observaron los fenómenos en un ambiente natural. Además, el objetivo principal es hallar la relacion entre dos variables estilos de afrontamiento y bienestar psicológico, en los alumnos de psicología de la Universidad Católica de Santa María de Arequipa (Hernández et al., 2014).

\section{Participantes}

La población la conforman todos los alumnos de la Escuela de Psicología de la Universidad Catolica de Santa Maria en Arequipa. Los criterios de inclusión de la muestra fueron: ser estudiante de Psicología de primer y/o segundo año, y ser menor de 19 años. Los criterios de exclusion fueron: personas con déficits visuales y auditivos, participantes que consuman sustancias psicoactivas, que tengan problemas familares, que no cumplan con la edad o que no deseen participar. 
Para seleccionar la muestra se hizo un muestreo no probabilistico por oportunidad, que está formado por los sujetos disponibles a los cuales tuvimos acceso (Hernández et al., 2014). Entonces la muestra quedó conformada por 120 alumnos pertenecientes a la Escuela de Psicología de la Universidad Católica de Santa María. De acuerdo a la información obtenida de los datos personales la edad promedio es de 19 años y desviación estándar de \pm 1.536 , de los cuales el $68 \%(n=82)$ son del sexo femenino y el $32 \%(n=38)$ de sexo masculino.

\section{Instrumentos}

Se usó el Cuestionario de Afrontamiento del Estrés (CAE) que es una medida de autoinforme diseñada para evaluar siete estilos básicos de afrontamiento: focalizado en la solución del problema, autofocalización negativa, reevaluación positiva, expresión emocional abierta, evitación, búsqueda de apoyo social y religión. A partir de una muestra de estudiantes universitarios $(\mathrm{N}=592)$ los resultados demostraron una clara estructura factorial de siete factores que representaban los siete estilos básicos de afrontamiento. Las correlaciones entre los factores fueron bajas o moderadas. Los coeficientes de fiabilidad de Cronbach para las 7 subescalas variaron entre 0.64 y $0.92(\mathrm{M}=0.79)$ (Sandin \& Chorot, 2003). Los resultados sobre la estructura del afrontamiento sugieren que las 7 dimensiones aisladas son bastante consistentes y confieren validez y confiabilidad a las 7 subescalas del CAE.
Para evaluar el bienestar psicológico se utilizó la Escala de Bienestar Psicológico (BIEPS-J), creada por Casullo y Castro, y adaptada por Casullo, Brenlla, Castro, Gonzáles, Maganto y Morote (2002). Posee 13 ítems, los cuales tienen por finalidad evaluar el bienestar psicológico. Los usuarios, deben responder en función a lo que sintieron y pensaron durante el último mes. Este instrumento tiene tres elecciones de respuesta: 1) "De acuerdo" (3 puntos); 2) "Ni de acuerdo ni en desacuerdo" (2 puntos) y 3) "En desacuerdo" (1 punto). La escala posee cuatro factores: Vínculos psicosociales, Aceptación de sí mismo, Control de situaciones y Proyectos. La suma de los ítems es la puntuación directa. Al puntaje total se asigna un valor ubicado en los percentiles del instrumento. Puntajes inferiores a 25 revelan que el sujeto se percibe con un bajo bienestar psicológico comprometiendo diversos aspectos importantes. Puntajes mayores a 50 o 75 definen un bienestar promedio o alto, lo cual hace referencia a sujetos satisfechos o muy satisfechos con su vida, en términos generales.

Para la validez y confiabilidad de la escala se hizo un estudio con una muestra de Lima que consistía en 413 jóvenes que pertenecen a instituciones particulares y nacionales, y se realizó un análisis factorial para identificar la validez del instrumento. Los resultados indicaron que los cuatro factores se confirman con los ítems a excepción del ítem 2 que no se ubica en ninguna área y el to que se ubica en el factor Vínculos cuando originalmente 
perteneceal factor Aceptación. Al calcular la confiabilidad mediante la prueba alfa de Cronbach se obtuvo una puntuación superior a .7 para cada una de los factores. El $70 \%$ de las correlaciones ítem-test obtuvieron una puntuación superior a .20 y los que se encuentran por debajo estuvieron muy cercanos al nivel esperado

\section{Procedimientos}

Se procedió a solicitar los permisos respectivos a la escuela de la Escuela de Psicología de la Universidad Católica de Santa María de Arequipa, indicando la intención de realizar una investigación con los alumnos de primer y segundo año, para conocer sus estilos de afrontamiento y el bienestar psicológico. Una vez concedido el permiso, se coordinó con los profesores para no interferir en labores académicas. Luego se procedió a la evaluación de los alumnos con las pruebas respectivas, previamente entregado un consentimiento informado; teniendo en cuenta los criterios de inclusión y exclusión mencionados anteriormente, así como las indicaciones necesarias para la aplicación de cada prueba.

\section{Análisis estadístico}

Luego de la recolección de datos, se procedió a la organización y corrección de los instrumentos para ingresarlos en el programa estadístico SPSS versión 23 y calcular los estadísticos descriptivos e inferenciales, aplicando la prueba de correlación de Pearson, porque permite medir el grado de relación de dos variables siempre y cuando ambas sean cuantitativas y continuas, como lo son en este caso.

\section{Resultados}

Con respecto a los estilos de afrontamiento, en la Figura 1 se muestra que existe una preferencia por el estilo de afrontamiento de Revaluación positiva con un $22 \%$ ( $\mathrm{f}=26$ ), y le siguen la Focalización en la solución del problema y Búsqueda de apoyo social" con $18 \%(f=21)$ cada una. Después está el estilo de Evitación con $15 \%$ $(\mathrm{f}=18)$, luego le sigue el estilo Expresión emocional abierta con $12 \%$ ( $f=14$ ), y el estilo de Autofocalización negativa con $11 \%(\mathrm{f}=13)$, y por último está el estilo de afrontamiento basado en la Religión con un $4 \%(f=7)$. 
Figura 1. Porcentaje de los estilos de afrontamiento

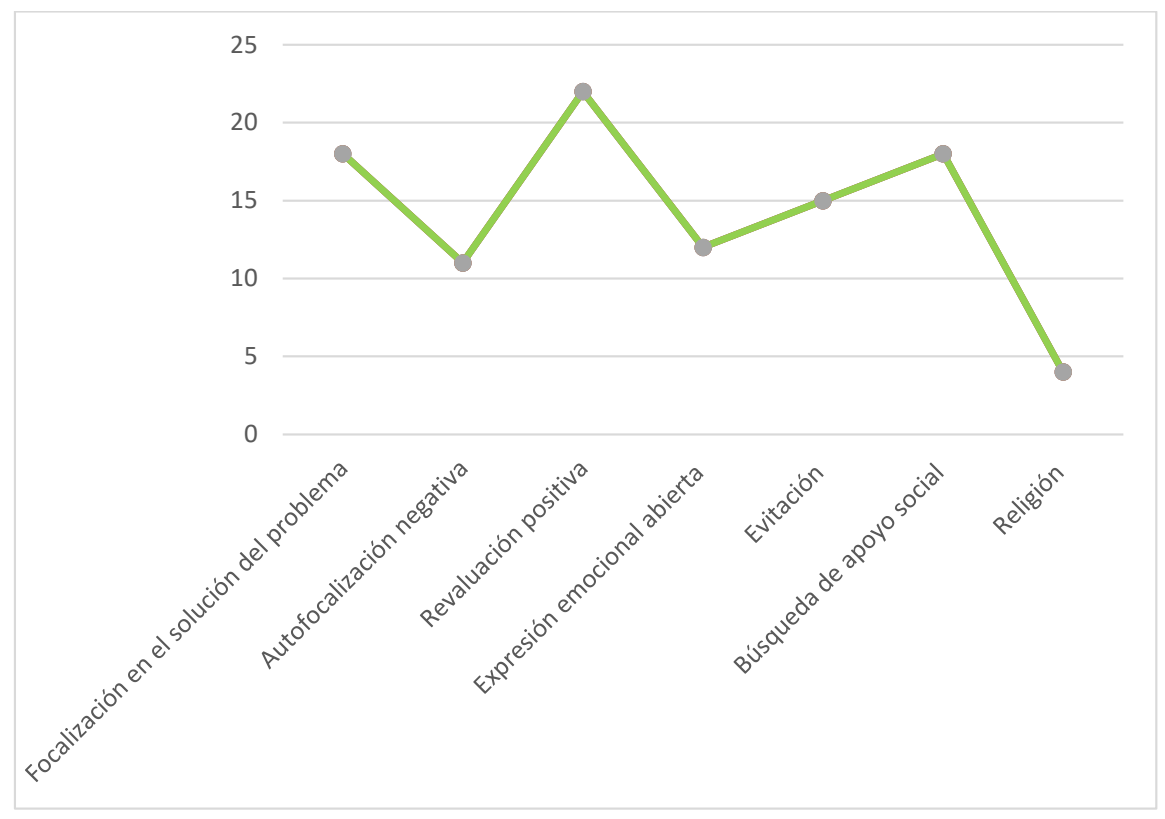

Por otra parte, el nivel de bienestar de los alumnos los primeros años de la carrera de psicología de la Universidad Católica de Santa María, registra una puntuación promedio para el análisis de la Escala de Bienestar Psicológico (BIEPS). A partir de esta puntuación se dividióla muestra en dos grupos: Alto Bienestar: los que presentaron en sus puntuaciones un percentil mayora 75; y Bajo Bienestar:que comprendepuntuaciones con un percentil menor a 25 . El $84 \%$ ( $\mathrm{f}=$ 101) de la muestra presentó un alto bienestar y solo el $4 \%(f=5)$ obtuvo un bajo nivel de bienestar psicológico, sobreentendiendo que el $12 \%(\mathrm{f}=14)$ estuvo dentro del promedio (Figura 2). 
Figura 2. Porcentaje de los niveles de bienestar psicológico

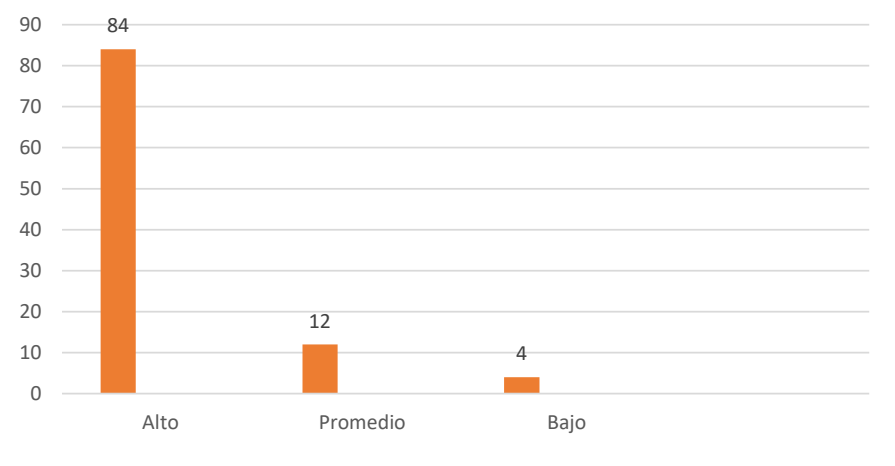

Seguidamente, se realizó un análisis de estudiantes de Psicología que autopercorrelación entre el bienestar psicológicoy ciben un mayor bienestar psicológico los estilos de afrontamiento de la muestra, utilizan técnicas de afrontamiento de para así responder a nuestra pregunta de focalización en la solución del problema, investigación. En la Tabla 1 se muestran revaluación positiva y búsqueda deapoyo los resultados obtenidos, de modo que social; ya que presentan correlaciones podemos decir que nuestra muestra de significativas positivas bajasy moderadas.

Tabla 1. Correlación entre los estilos de afrontamiento y el bienestar psicológico

\begin{tabular}{|lc|}
\hline Estilo de Afrontamiento & Bienestar psicológico \\
\hline Focalización en el solución del problema & $.332^{* *}$ \\
Autofocalización negativa & 0.089 \\
Revaluación positiva & $.194^{*}$ \\
Expresión emocional abierta & -0.097 \\
Evitación & -0.042 \\
Búsqueda de apoyo social & $.215^{*}$ \\
Religión & -0.012 \\
\hline$* * \mathbf{p}<.01{ }^{*} \mathbf{p}<.05$ & \\
\hline
\end{tabular}

Luego se correlacionaron los distintos se aprecia en la Tabla 2, en la que la estilos de afrontamiento con las dimen- dimensión de Aceptación de sí mismo siones del bienestar psicológico como tiene 4 correlaciones, tres positivas con 
los estilos de Focalización en la solución del problema, Revaluación positiva y de Búsqueda de apoyo social, y una negativa con Autofocalización negativa. En la dimensión de Control de situaciones vemos correlaciones positivas con Focalización en la solución del problema y Revaluación positiva, y una correlación negativa de nuevo con Autofocalización negativa. La situación parece ser similar con la dimensión de Vínculos psicosociales, donde se aprecian correlaciones positivas con la Focalización en la solución del problema, la Revaluación positiva y la Búsqueda deapoyo social,y una correlación negativa con Autofocalización. Por último, en la dimensión Proyectos vemos solo dos correlaciones, la positiva con Focalización en la solución de problemas y la negativa con Autofocalización negativa.

\section{Tabla 2. Correlación entre los estilos de afrontamiento y las dimensiones de bienestar psicológico}

\begin{tabular}{|lcccc|}
\hline \multicolumn{1}{|c}{ Estilo de Afrontamiento } & $\begin{array}{c}\text { Aceptación } \\
\text { de sí mismo }\end{array}$ & $\begin{array}{c}\text { Control } \\
\text { de situaciones }\end{array}$ & $\begin{array}{c}\text { Vínculos } \\
\text { psicosociales }\end{array}$ & Proyectos \\
\hline Focalización en el solución del problema & $.283^{* *}$ & $0,380^{* *}$ & $.0183^{*}$ & $.244^{* *}$ \\
Autofocalización negativa & $-0.24^{* *}$ & $-.183^{*}$ & -0.141 & $-.320^{* *}$ \\
Revaluación positiva & $.216^{*}$ & $.237^{* *}$ & $.210^{*}$ & 0.065 \\
Expresión emocional abierta & -0.017 & -0.112 & -0.026 & -0.109 \\
Evitación & -0.036 & -0.034 & -0.06 & -0.065 \\
Búsqueda de apoyo social &, $263^{* *}$ & 0.173 & $.191^{*}$ & 0.161 \\
Religión & -0.023 & -0.063 & -0.044 & 0.075 \\
\hline
\end{tabular}

${ }^{* *} \mathbf{p}<.01{ }^{*} \mathbf{p}<.05$

\section{Discusión}

El objetivo de esta investigación era determinar la relación entre los estilos de afrontamiento y el bienestar psicológico de los alumnos de psicología de la Universidad Católica de Santa María de Arequipa, y el principal hallazgo indica que hay una relación estadisticamente significativa entre tres estilos de afrontamiento y un alto bienestar psicológico.

Los jóvenes con un elevado nivel de bienestar psicológico se caracterizaron por analizar la causa de los problemas de un modo sistemático y tomaron en cuenta diferentes puntos de vista para solucionarlos, lo que muestra un modelo sistematico de actuar, para conseguir resultados concretos en corto tiempo (Focalización en la solución del problema). Igualmente pusieron de manifiesto la capacidad para ver los aspectos positivos delas distintassituacionesadversas, loque puedellevara los estudiantesa pensar que todo tiene una solución y que hay cosas más importantes (Revaluación positiva). Al mismo tiempo, se destacó la habilidad de búsqueda deapoyosocial quehace referencia a la posibilidad de compartir los problemas con otrosy conseguir ayuda para resolverlos. 
Si se analizan con profundidad estas tres estrategias, se puede afirmar que tienen en común la búsqueda de soluciones concretas y sin gran desgaste cognitivo ni emocional (Sandín \& Chorot, 2003).

Nuestros resultados concuerdan con los de Quiros (2013) y Tacca y Tacca (2019) que encontraron que el estilo de afrontamiento más usado es el enfocado en el problema, seguidoporlaemocióny, finalmente, el evitativo. Estos resultados también concuerdan con que los jóvenes buscan la reinterpretación positiva, la planificación, el afrontamientoactivoy la búsquedadesoportesocial.

La explicación a estos resultados puedeestar en las características deetapa evolutiva escogida, ya que los jóvenes de esta edad, le dan mayorimportanciaa suafirmación personal, a las relaciones con sus paresy a su sociabilidad, que son aspectos asociados a su grado de bienestar (Martínez et al., 2006).

También podemos explicarlo debido a los nuevos enfoques de la juventud de Solis y Vidal (2006) que refieren que el individuo desarrolla dichas capacidades frentea situaciones estresantes dependiendo de su nivel aprendizaje y conocimientos, además de tener un adecuado manejo pensamientos y emociones frente a las adversidades. Esto permitiría un apropiado desarrollo de sus habilidades organizativas y planificadoras para llegara una solución frenteal problema (estilo focalizadoen la solución del problema y revaluación positiva). Si consideramos que nuestra muestra estaba conformada por estudiantes universitarios de Psicología es lógico suponer que poseen un buen nivel de aprendizaje y conocimientos referentes al manejo de estrés, a pesar de encontrarse en los primeros años de estudio.

Para explicar la relación entre búsqueda de apoyo social y bienestar, podemos recurrir a San Juán y Magallanes (2007), quienes nos dicen que los jóvenes de hoy se proponen adquirir independencia de las figuras parentales, yen esa postura, el grupodepares constituye una red de apoyo social esencial. De allí es que este vínculo sea, probablemente, el más importante para los afectos, la competencia, la solidaridad, etc. Aspectos quevan a exigir estrategias de afrontamiento que si no son exitosas generan angustia. El empleo de esta estrategia da cuenta de que el joven ha aprendido que es más factible solucionar sus problemas con la ayuda de una red social.

En el análisis por dimensiones vemos que el estilo de focalización en la solución del problema se relaciona positivamente con todas las dimensiones, lo que nos indica que nuestra muestra es capaz de aceptarse a sí misma, incluyendo lo bueno y lo malo. También sugiere que tienen buena percepción del control de situaciones, ya que son capaces de crear y modelar los contextos para adecuarlos a sus necesidades personales. Al mismo tiempo, son capaces de crear vínculos psicosociales estables con calidez, confianza y capacidad empática y afectiva. Por último, nos revela que tienen facilidad para fijar metas y proyectos de vida, ya que son capaces de darle un significado a sus experiencias (Sandín \& Chorot, 2003).

El estilo de revaluación positiva se relaciona positivamente con todas las dimensiones del bienestar psicológico, menos con la 
dimension de proyectos, lo que puede ser un indicador de que las personas con este estilo de afrontamiento no se preocupan mucho por el futuro, ya que se centran en el presente, identificando losaspectos positivos para tolerar la problemáticaygenerarpensamientos positivos que favorezcan enfrentar las situaciones negativas (Lee-Baggley \& Preece, 2005).

El estilode búsqueda deapoyo social, muestra correlaciones positivas con la aceptación de sí mismo y los vínculos psicosociales, lo cual tiene bastante lógica, porque para poder confiar y pedir ayuda a terceros, se debe que tener mínimamente un vínculo afectivo positivo. Loque sí resulta intersante es la no correlación con las dimensiones control de las situacionesy proyectos, lo que podemos intentar explicar con la teoria del locus de control externo, que según estudios de Azozollini y Bail (2010), las personas que tienen este estilo atribuyen sus problemas a causas externasybuscan laautoconfirmación deterceros. Entonces, estas personas cuando se enfrentan a situaciones estresantes las atribuyen a causas externas y buscan apoyo social para que le den apoyo emocional y confirmen sus ideas. Y para explicar la baja correlación en proyectos, podemos tomar la teoría autoeficacia en estudios de Borzone (2017) queexplica que las personas queusan este estilo, lo hacen porque no se sienten capaces de resolver una situación estresante por sí solos, por lo que buscan apoyo, entonces como no confian en sus propias capacidades les cuesta proyectarse a futuro.

Lo que resulta llamativo de las correlaciones por dimensiones son los resultados del estilo de autofocalización negativa, que muestra correlaciones negativas con todas las dimensiones del bienestar psicológico, lo que nos dice que a menor bienestar habrá un mayor estilo autofocalizado negativo. Esto se puede dar porque esta estrategia esta centrada en la emoción,entoncesestáacompañadadefuertes tendenciasacatastrofizar,yseleharelacionado consíntomasdepresivos, menorpercepción de apoyosocial, ansiedad, percepcióndemalestar psicológico y detrimento en la calidad devida percibida (Griswold et al., 2005).

Si nos preguntamos por qué no hay relación entre el bienestar psicológico con los otros estilos de afrontamiento, ya sea de forma general o por dimensiones (autofocalización negativa, expresión emocional negativa, evitación y religión); podemos intentar explicarlo medinate Frydenberg y Lewis (1997), quienes explican que estos mecanismos requieren más desgaste cognitivo y emocional, cosa que los jóvenes no buscan. Haciendo un análisis desde la polaridad activo-pasivo (Millon, 1998), sepuedeseñalar queesta posición pasiva ante las situaciones, indudablemente es de riesgo para el joven, pues la resolución exitosa decualquier hecho vital implica poner en marcha estrategias cognitivas y afectivas que, con principio de realidad conduzcan a modificar la situación problemática planteada, asumiendo una posición activa con soluciones concretas y rápidasqueno requieran de muchodesgaste.

A modo de resumen se puede afirmar que la mayoria dejovenes estudiados ( $84 \%$ ) presentaron un elevado nivel debienestarpsicológico yque utilizaron preferentemente estrategias deafrontamientodetipoactivocomoconcentrarse en resolver el problema, fijarse en lo positivoy buscarapoyo social. En cambio los 
que presentaron bajo bienestar psicológico emplearonestrategiasquesepuedencalificar como pasivas, tales como autoinculparse, ignorar el problemay falta deafrontamiento. Delos resultadosobtenidossurgen otras interrogantes acerca de quéfactores determinan quealgunos estudiantesempleen determinadasestrategiasyotrosotras, según la diferencia de género o por edades.

\section{Financiamiento}

La presente investigación fue autofinanciada.

\section{Conflictos de interés}

La autora declara que no tiene conflictos de interés. 


\section{Referencias}

Azzollini, S., \& Bail, V. (2010). La relación entre el locus de control, las estrategias de afrontamiento y las prácticas de autocuidado en pacientes diabéticos. Anuario de Investigaciones, 26(1), 287-297.

Borzone, M. (2017). Autoeficacia y vivencias académicas en estudiantes universitarios. Acta Colombiana de Psicología, 20(1), 266-274. https://doi.org/10.14718/ ACP.2017.20.1.13

Casullo, M., \& Castro, A. (200o). Evaluación del bienestar psicológico en estudiantes adolescentes argentinos. Revista de Psicología (Pontificia Universidad Católica del Perú), 18(2), 35-68.

Cornejo, M., E Lucero, M. (2005). Preocupaciones vitales en estudiantes universitarios relacionado con bienestar psicológico y modalidades de afrontamiento. Fundamentos en Humanidades, 6(2), 143-153. https://www.redalyc.org/pdf/184/18412608.pdf

Cuadra, H., \& Florenzano, R. (2003) El bienestar subjetivo: Hacia una Psicología Positiva. Revista de Psicología de la Universidad de Chile, 12(1), 83-96. https://doi. org/10.5354/0719-0581.2012.17380

Gómez, V., Villegas, C., Barrera, F., \& Cruz, J. (2007). Factores predictores de bienestar subjetivo en una muestra colombiana. Revista Latinoamericana de Psicología, 39, $311-325$.

González, R., Montoya, I., Casullo, M., E Bernabéu, J. (2002). Relación entre estilos y estrategias de afrontamiento y bienestar psicológico en adolescentes. Psicothema, 14(2), 363-368. http://www.psicothema.com/pdf/733.pdf

Griswold, G., Evans, S., Spielman, L., \& Fishman, B. (2005). Coping Strategies of HIV Patients with Peripheral Neuropathy. AIDS Care, 17, 711-720.

Frydenberg, E., E Lewis, R. (1997). ACS. Escalas de Afrontamiento para adolescentes (Manual adaptación Española). TEA.

Hernández, R., Fernández, C., E Baptista, P. (2014). Metodología de la investigación. 6ta ed. McGraw-Hill.

Lazarus, R., E Folkman S. (1984). Estrés y procesos cognitivos. Ediciones Martínez Roca. 
Lee-Baggley, D., \& Preece, M. (2005). Coping with interpersonal stress: Role of big five traits. Journal of Personality, 73(5), 1141-1180. https://doi. org/10.1111/j.1467-6494.2005.00345.x

Macías, A., Orozco, C., Amarís, M., \& Zambrano, J. (2013). Estrategias de afrontamiento individual y familiar frente a situaciones de estrés psicológico. Psicología desde el Caribe, 30(1), 123-145.

Marsollier, R., E Aparicio, M. (2011). El bienestar psicológico en el trabajo y su vinculación con el afrontamiento en situaciones conflictivas. Psicoperspectivas, 10(1), 209-220.

Martínez-Correa, A., Reyes del Paso, G., García-León, A., E González-Jareño, M. I. (2006). Optimismo/pesimismo disposicional y estrategias de afrontamiento del estrés. Psicothema, 18(1), 66-72.

Matalinares, M., Díaz, G., Ornella, R., Baca, D., Uceda, J., E Yaringaño, J., (2016). Afrontamiento del estrés y bienestar psicológico en estudiantes universitarios de Lima y Huancayo. Persona, 19, 105-126.

Millon, T. (1998). Los trastornos de la personalidad más allá del DSM IV. Masson.

Omar, A., Paris, L., Aguiar, M., Almeida, S., E Del Pino, R. (2009). Validación del inventario de bienestar subjetivo con muestras de jóvenes y adolescentes argentinos, brasileros y mexicanos. Suma Psicológica, 16, 69-84.

Organización Mundial de la Salud. (2013). Salud mental: un estado de bienestar. https://www.who.int/features/factfiles/mental_health/es/

Quiros, S. (2013). Burnout y afrontamiento en un grupo de agentes de tráfico del aeropuerto. (Tesis de pregrado). Pontificia Universidad Católica del Perú, Lima, Perú. http://tesis.pucp.edu.pe/repositorio/handle/123456789/5098

Romero, A., Zapata, R., García-Mas, A., Brustad, R. J., Garrido, R., \& Letelier, A. (2010). Estrategias de afrontamiento y bienestar psicológico en jóvenes tenistas de competición. Revista de Psicología del Deporte, 19(1), 117-133. https://www. rpd-online.com/article/view/66o

Sandín, B., \& Chorot, P. (2003). Cuestionario de Afrontamiento del Estrés (CAE) Desarrollo y Validación Preliminar. Revista de Psicopatología y Psicología Clínica, $8,29-54$. 
Sanjuán, P., \& Magallares, P. (2007), Estilos explicativos y estrategias de afrontamiento. Revista Clínica y Salud, 18(1), 83-98. http://www.redalyc.org/articulo. oa?id=180613874006

Solís, M., \& Vidal, M. (2006). Estilos y estrategias de afrontamiento en adolescentes. Revista de Psiquiatría y Salud Mental Hermilio Valdizan, 7(1), 33-39.

Tacca, D., \& Tacca, A. (2019). Estilos de afrontamiento y bienestar psicológico en estudiantes de postgrado. Revista de Psicologia, 21(1), 23-32.

Urbano, D. (2019). Estilos de afrontamiento al estrés y bienestar psicológico en adolescentes de la escuela de líderes escolares de Lima Norte - 2017. (Tesis de grado). Universidad Peruana Cayetano Heredia, Lima, Perú.

Urrelo, A., \& Huamani, J. C. (2019). Satisfacción con la vida y estilos de afrontamiento en adolescentes de colegios públicos en la ciudad de Arequipa. Revista de Psicología (Universidad Católica San Pablo), 9(2), 13-32.

Vallejo, L. (2007). Bienestar subjetivo y estrategias de afrontamiento en estudiantes del Colegio Perpetuo Socorro del distrito de Trujillo. (Tesis de grado). Universidad César Vallejo, Trujillo, Perú.

Recibido: 28 de junio de 2020

Revisado: 12 de diciembre de 2020

Aceptado: 11 de marzo de 2021 